\title{
Color Transparency and Hadron Formation Effects in High-Energy Reactions on Nuclei
}

\author{
Alexei Larionov ${ }^{1,2, *}$ and Mark Strikman ${ }^{3}$ \\ 1 Institut für Theoretische Physik, Universität Giessen, D-35392 Giessen, Germany \\ 2 Frankfurt Institute for Advanced Studies (FIAS), D-60438 Frankfurt am Main, Germany \\ 3 Department of Physics, Pennsylvania State University, University Park, PA 16802, USA; mxs43@psu.edu \\ * Correspondence: larionov@fias.uni-frankfurt.de
}

Received: 15 December 2019; Accepted: 13 January 2020; Published: 17 January 2020

\begin{abstract}
An incoming or outgoing hadron in a hard collision with large momentum transfer gets squeezed in the transverse direction to its momentum. In the case of nuclear targets, this leads to the reduced interaction of such hadrons with surrounding nucleons which is known as color transparency $(\mathrm{CT})$. The identification of $\mathrm{CT}$ in exclusive processes on nuclear targets is of significant interest not only by itself but also due to the fact that $\mathrm{CT}$ is a necessary condition for the applicability of factorization for the description of the corresponding elementary process. In this paper we discuss the semiexclusive processes $A\left(e, e^{\prime} \pi^{+}\right), A\left(\pi^{-}, l^{-} l^{+}\right)$and $A\left(\gamma, \pi^{-} p\right)$. Since CT is closely related to hadron formation mechanism, the reduced interaction of 'pre-hadrons' with nucleons is a common feature of generic high-energy inclusive processes on nuclear targets, such as hadron attenuation in deep inelastic scattering (DIS). We will discuss the novel way to study hadron formation via slow neutron production induced by a hard photon interaction with a nucleus. Finally, the opportunity to study hadron formation effects in heavy-ion collisions in the NICA regime will be considered.
\end{abstract}

Keywords: Glauber and Giessen Boltzmann-Uehling-Uhlenbeck (GiBUU) models; formation length; semiexclusive processes; ultraperipheral and central heavy ion collisions; $n, p, \pi$ and $\Lambda+\Sigma^{0}$ production

\section{Introduction}

Hard processes, e.g., exclusive meson electroproduction with $Q^{2} \gg 1 \mathrm{GeV}^{2}$, can be only described by taking into account quark-gluon degrees of freedom. The characteristic transverse size of the incoming and outgoing color-neutral quark configurations in a hard process is $r_{t} \sim 1 / Q$ and, thus, they can be regarded as point-like configurations (PLCs). It can be shown within PQCD [1] that the interaction cross section of the small- $r_{t}$ color singlet $q \bar{q}$ pair and a proton behaves geometrically at $r_{t} \rightarrow 0$, i.e., $\sigma_{q \bar{q}} \propto r_{t}^{2}$. Therefore, the interaction of PLCs with surrounding nucleons in the nuclear target is strongly reduced which is known as the CT phenomenon, see [2] for the most recent review of $\mathrm{CT}$.

A PLC is not an eigenstate of the QCD Hamiltonian and, therefore, it is unstable and expands to the normal hadronic size on the proper time scale $\lesssim 1 \mathrm{fm} / \mathrm{c}$. However, the expansion time of the PLC can be large due to the Lorentz time dilation. It is thus possible to observe $\mathrm{CT}$ if the incoming and/or outgoing PLCs are fast enough in the nuclear target rest frame.

At ultrarelativistic energies, where the PLCs are practically 'frozen', CT has been observed at Fermilab [3] in coherent diffractive dissociation of a $500 \mathrm{GeV} / \mathrm{c}$ pion in a pair of high- $k_{t}$ jets on nuclear targets following theoretical predictions [4]. The smallness of initial- and final-state interactions (ISI,FSI) has been concluded from the mass number dependence of the cross section 
$A^{\alpha}, \alpha=1.6$ at $k_{t} \gtrsim 1 \mathrm{GeV}$ (which is far away from expected $\alpha=2 / 3$ for soft coherent diffraction but agrees with calculations of refs. $[4,5])$.

At intermediate energies $\left(E_{\text {beam }} \sim 10 \mathrm{GeV}\right)$, CT becomes less pronounced. (The beam energy at which $\mathrm{CT}$ will be observable depends, of course, on the concrete process. The minimum requirement for $\mathrm{CT}$ is that at least one hadron participating in the hard process should be fast. This can be either an incoming or an outgoing hadron. The momentum transfer from the beam particle to the outgoing hadrons is shared between them so that the largest possible value is given by the beam momentum.) This can be understood from the decomposition of the wave function of a PLC in a hadronic basis of states with fixed momentum $p_{h}$ (that is the momentum of the genuine hadron ' $h$ ' to which the PLC is asymptotically converted):

$$
\left|\Psi_{P L C}(t)>=\sum_{i=1}^{+\infty} a_{i} e^{-i E_{i} t}\right| \Psi_{i}>=e^{-i E_{1} t} \sum_{i=1}^{+\infty} a_{i} e^{i\left(E_{1}-E_{i}\right) t} \mid \Psi_{i}>, \quad E_{i}=\sqrt{p_{h}^{2}+m_{i}^{2}} .
$$

Due to different phase velocities, $E_{i} / p_{h}$, of the plane waves the initially compact in space configuration expands on the length scale of the order of

$$
l_{h}=\frac{1}{E_{2}-E_{1}} \simeq \frac{2 p_{h}}{\Delta M^{2}}
$$

where $\Delta M^{2}=m_{2}^{2}-m_{1}^{2}$ assuming the relativistic limit, $p_{h} \gg m_{1}, m_{2}$. Equation (2) can be thus regarded as an estimate of the hadron $1(\equiv h)$ formation (or coherence) length. The hadronic state 2 is the first radially excited state of the hadron 1 . Hence, we can estimate $\Delta M^{2} \simeq m_{N^{*}(1440)}^{2}-m_{N}^{2} \simeq 1.2 \mathrm{GeV}^{2}$ for the nucleon. However, for the pion that has a Goldstone nature the same argument does not apply. Thus, assuming that the quark and antiquark each carry $1 / 2$ of the light cone (LC) momentum of a $q \bar{q}$ system we estimate $\Delta M^{2} \simeq 4\left(m_{q}^{2}+\left\langle k_{t}^{2}\right\rangle\right)-m_{\pi}^{2} \simeq 0.93 \mathrm{GeV}^{2}$ for the pion, where $m_{q}=0.340 \mathrm{GeV}$ is the constituent quark mass and $\left\langle k_{t}^{2}\right\rangle^{1 / 2} \simeq 0.35 \mathrm{GeV} / \mathrm{c}$ is the average transverse momentum of a quark in a hadron [6].

These estimates are in a reasonable agreement with the empirical range obtained from the analysis of pionic nuclear transparency at JLab [7], $\Delta M^{2} \simeq 0.7-1.1 \mathrm{GeV}^{2}$ corresponding to

$$
l_{h}=0.4-0.6 \mathrm{fm} \frac{p_{h}}{\mathrm{GeV} / \mathrm{c}},
$$

At $p_{h} \sim 10 \mathrm{GeV} / \mathrm{c}$, the empirical pion formation length (3) becomes comparable with the radii of heavy nuclei indicating the onset of CT.

At intermediate energies, clear CT signals have been experimentally observed from the $Q^{2}$-dependence of nuclear transparency in the electroproduction of a pion $A\left(e, e^{\prime} \pi^{+}\right)$for $Q^{2}=1-5 \mathrm{GeV}^{2}[8]$ and of a $\rho$-meson $A\left(e, e^{\prime} \rho^{0}\right)$ for $Q^{2}=0.8-2.4 \mathrm{GeV}^{2}$ [9] at JLab. However, CT has not been observed for the quasielastic proton electroproduction $A\left(e, e^{\prime} p\right)$ studied at SLAC and JLab. (Squeezing proton probably needs larger $Q^{2}$ values than for pion.)

$\mathrm{CT}$ has been predicted for the hadron-induced semi-exclusive processes with large momentum transfer $h+A \rightarrow h+p+(A-1)^{*}[10,11]$. So far, only $\mathrm{C}(\mathrm{p}, 2 \mathrm{p})$ process at $\Theta_{c . m .}=90^{\circ}$ has been studied experimentally at BNL [12]. The nuclear transparency for this process increases with beam momentum until $p_{\text {lab }} \sim 9 \mathrm{GeV} / \mathrm{c}$ in agreement with CT, but then it starts to decrease. In [13], such a complex behavior has been explained by the intermediate (very broad, $\Gamma \sim 1 \mathrm{GeV}$ ) $6 q c \bar{c}$ resonance formation with mass $\sim 5 \mathrm{GeV}$. Alternatively in [14], the same behavior has been explained by stronger absorption of the large-size quark configurations produced by the Landshoff mechanism (three-gluon exchange).

In the inclusive processes at high energies, e.g., in DIS, the formation of PLCs is less clear, since even at high $Q^{2}$ the momentum transfer is shared between many particles. Nevertheless, most theoretical studies of DIS off nuclei include CT effects for the interaction of fast pre-hadrons with nuclear medium using dynamical hadron formation models [15-17]. Hadron 
formation effects are included in microscopic transport models for high-energy heavy ion collisions, such as UrQMD [18], HSD [19], and the GiBUU model [20].

The purpose of this paper is to elucidate the effect of PLC expansion on CT. We start from the most clean exclusive processes which can be described on the basis of the Glauber model supplemented by the quantum diffusion effect [6]. Then we continue discussing the effects of hadron formation on slow neutron production in photon-nucleus interactions. Finally, we address proton, pion and hyperon rapidity and $p_{t}$ spectra in $\mathrm{pA}$ - and central AA collisions.

The structure of the paper is as follows. In Section 2 we present the results of the Glauber model and the quantum diffusion model (QDM) calculations of the nuclear transparency in pion electroproduction $A\left(e, e^{\prime} \pi^{+}\right)$at large $Q^{2}$, in pionic Drell-Yan process $A\left(\pi^{-}, l^{+} l^{-}\right)$at large invariant mass of the dilepton pair, and in large-angle pion photoproduction $A\left(\gamma, \pi^{-} p\right)$. In Section 3 the GiBUU model supplemented by the statistical multifragmentation model (SMM) for the decay of excited nuclear residue is applied to describe slow $(E<10 \mathrm{MeV})$ neutron production in high-energy virtual-photon-nucleus interactions. We study the sensitivity of the slow neutron production to various treatments of hadron formation. Section 4 contains the discussion of the results of the GiBUU calculations of the pA- and central AA collisions. Finally, in Section 5 we summarize our results and draw conclusions.

\section{Exclusive Processes}

There is a delicate connection between CT and pQCD-factorization, namely, if the latter is applicable for the description of some hard exclusive process then CT necessarily appears for that process in the nuclear target. This is because without CT the multiple gluon exchanges before and after the hard process would not be suppressed. Therefore, CT is important for testing the applicability of factorization in exclusive hard processes.

\subsection{Pion Electroproduction}

The process $A\left(e, e^{\prime} \pi^{+}\right)$at large space-like photon virtuality can be used to better understand the mechanism of the elementary $\gamma^{*} p \rightarrow \pi^{+} n$ transition. It is argued in ref. [21] that for the longitudinal photon the pion pole dominates, while for the transverse photon the quark-gluon degrees of freedom are important (PYTHIA/JETSET simulation). On the other hand, the factorization theorem [22] renders descriptions in terms of hadronic degrees of freedom to be questionable for the longitudinal photon. Having this uncertainty in mind, it is reasonable to assume that PLCs are formed both for longitudinal and transverse photon, i.e., in the non-polarized channel.

The experimental data [8] were taken in the collinear kinematics, $\mathbf{p}_{\pi} \| \mathbf{q}=\mathbf{p}_{e}-\mathbf{p}_{e^{\prime}}$. This leads to the following expression for the nuclear transparency ( $z$-axis is parallel to the pion momentum $\mathbf{p}_{\pi}$ ):

$$
T=\frac{d^{5} \sigma_{e A \rightarrow e^{\prime} \pi^{+}} / d^{3} p_{e^{\prime}} d \Omega_{\pi^{+}}}{Z d^{5} \sigma_{e p \rightarrow e^{\prime} \pi^{+} n} / d^{3} p_{e^{\prime}} d \Omega_{\pi^{+}}}=\frac{1}{Z} \int d^{3} r \rho_{p}(\mathbf{r}) \mathrm{e}^{-\int_{z}^{\infty} d z^{\prime} \sigma_{\pi N}^{\text {eff }}\left(p_{\pi}, z^{\prime}-z\right) \rho\left(\mathbf{b}, z^{\prime}\right)},
$$

where $\rho_{p}(\mathbf{r})$ and $\rho(\mathbf{r})$ are the proton and nucleon densities, respectively. In Equation (4), the expansion of the pionic PLC is accounted for within the QDM [6] in terms of the effective pion-nucleon cross section:

$$
\sigma_{\pi N}^{\text {eff }}\left(p_{\pi}, z\right)=\sigma_{\pi N}\left(p_{\pi}\right)\left(\left[\frac{z}{l_{\pi}}+\frac{n^{2}\left\langle k_{t}^{2}\right\rangle}{M_{\mathrm{CT}}^{2}}\left(1-\frac{z}{l_{\pi}}\right)\right] \Theta\left(l_{\pi}-z\right)+\Theta\left(z-l_{\pi}\right)\right),
$$

where $\sigma_{\pi N}\left(p_{\pi}\right)$ is the empirical total pion-nucleon cross section, $n=2$ is the number of valence quarks and antiquarks, $M_{\mathrm{CT}}^{2}$ is the CT scale, and $l_{\pi}$ is the pion formation length. In the hard interaction point, $z=0$, the effective cross section (5) is reduced by a factor $\propto M_{\mathrm{CT}}^{-2}$ as compared to the empirical total cross section $\sigma_{\pi N}\left(p_{\pi}\right)$. With increasing propagation distance $z$ from the interaction point the effective 
cross section grows linearly with $z$ and becomes equal to $\sigma_{\pi N}\left(p_{\pi}\right)$ for $z \geq l_{\pi}$. In the kinematics of the pion electroproduction the CT scale $M_{\mathrm{CT}}^{2}$ is given by $Q^{2}=-\left(p_{e}-p_{e}^{\prime}\right)^{2}$.

Figure 1 displays the nuclear transparency as a function of $Q^{2}$. The Glauber model results are obtained by replacing $\sigma_{\pi N}^{\text {eff }}\left(p_{\pi}, z\right) \rightarrow \sigma_{\pi N}\left(p_{\pi}\right)$ in Equation (4). We see that the Glauber model significantly underpredicts the transparency. The QDM with the formation length of Equation (2) with $\Delta M^{2}=0.7 \mathrm{GeV}^{2}$ is in a good agreement with data for all considered targets, except gold where $\Delta M^{2}=1.4 \mathrm{GeV}^{2}$ is closer to the data. In the considered kinematics the pion formation length varies in the interval $l_{\pi}=1.6-2.5 \mathrm{fm}$, i.e., it is comparable to the r.m.s. radii of light nuclei, ${ }^{12} \mathrm{C}$ and ${ }^{27} \mathrm{Al}$. Due to larger average nucleon density, the relative effect of $\mathrm{CT}$ is, however, stronger for heavier targets.
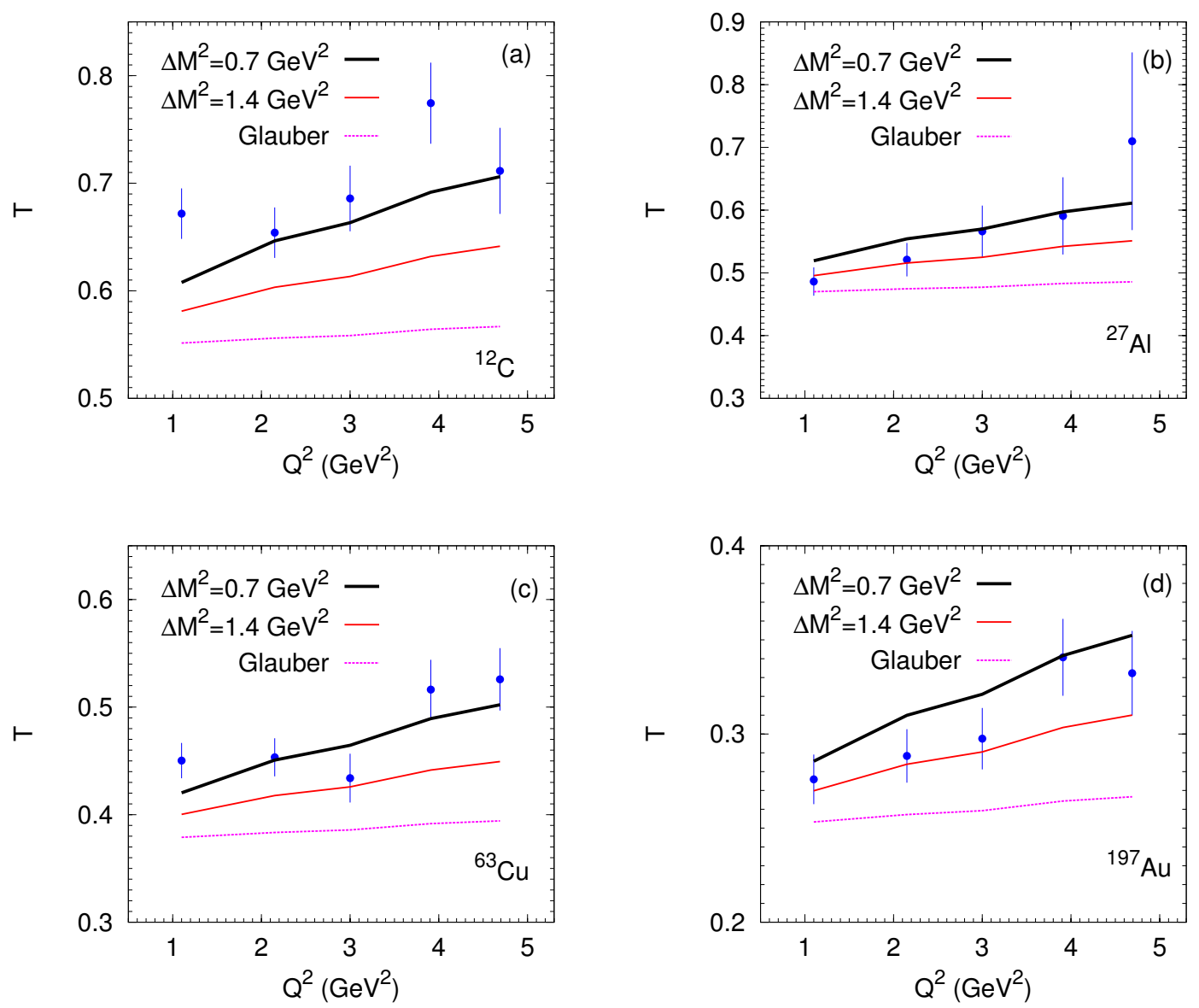

Figure 1. Transparency vs $Q^{2}$ for the $\left(e, e^{\prime} \pi^{+}\right)$reaction on the carbon (a), aluminum (b), copper (c), and gold (d) targets in the collinear kinematics. Dashed (magenta) line-Glauber model; thick (black) and thin (red) solid line—quantum diffusion model (QDM) with $\Delta M^{2}=0.7$ and $1.4 \mathrm{GeV}^{2}$, respectively. The pion momentum is $p_{\pi}=2.793,3.187,3.418,4.077$, and $4.412 \mathrm{GeV} / \mathrm{c}$ for $Q^{2}=1.10,2.15,3.00,3.91$ and $4.69 \mathrm{GeV}^{2}$, respectively, according to the kinematics of JLab experiment [8].

\subsection{Pionic Drell-Yan Process}

The process $\pi^{-} p \rightarrow l^{+} l^{-} n$ at $p_{\text {lab }}=15-20 \mathrm{GeV} / \mathrm{c}$ at small $|t|$ and large invariant mass of the dilepton pair, $M_{l^{+} l^{-}}$, has been proposed to study the generalized parton distributions of the nucleon at J-PARC $[23,24]$ (see also the feasibility study of W.C. Chang reported in [25]). Thus, the study of the nuclear transparency in the semiexclusive $A\left(\pi^{-}, l^{+} l^{-}\right)$process is complementary to the studies 
of factorization. The expression for the transparency has a similar form to Equation (4) except that the integration is done along the trajectory of the incoming pion (along $z$-axis):

$$
T=\frac{d^{4} \sigma_{\pi^{-} A \rightarrow l^{-} l^{+}} / d^{4} q}{Z d^{4} \sigma_{\pi^{-} p \rightarrow l^{-} l^{+} n} / d^{4} q}=\frac{1}{Z} \int d^{3} r e^{-\int_{-\infty}^{z} d z^{\prime} \sigma_{\pi N}^{\text {eff }}\left(p_{\pi}, z-z^{\prime}\right) \rho\left(\mathbf{b}, z^{\prime}\right)} \rho_{p}(\mathbf{r}),
$$

where $q=p_{l^{-}}+p_{l^{+}}-p_{\pi}$ is the four momentum transfer from the nucleus to the dilepton pair. The effective pion-nucleon cross section, $\sigma_{\pi N}^{\text {eff }}\left(p_{\pi}, z\right)$, is given by Equation (5) with $M_{\mathrm{CT}}^{2}=M_{l^{+} l^{-}}^{2}$. The selection of the exclusive transition $\pi^{-} p \rightarrow l^{+} l^{-} n$ in the nucleus can be done either by restricting the longitudinal momentum transfer $q^{z}$ for fixed $q_{t}$ and $M_{l^{+} l^{-}}^{2}$ [26] or directly applying the missing mass method [25].

Figure 2 shows the transparency for the pionic Drell-Yan process as a function of $p_{\text {lab }}$. The relative effect of CT grows with beam momentum due to increasing pion formation length and reaches $\sim 50-100 \%$ at $p_{\text {lab }}=20 \mathrm{GeV} / \mathrm{c}$. The effect is stronger for heavier targets. It is, however, interesting that in the calculation with CT the nuclear transparency reaches saturation at $p_{\mathrm{lab}}=15-20 \mathrm{GeV} / \mathrm{c}$ for light targets, ${ }^{12} \mathrm{C}$ and ${ }^{27} \mathrm{Al}$, while it continues to increase with $p_{\text {lab }}$ for the heavier ones. This behavior is explained by the approximate relation $l_{\pi} \sim 2 R$ which is fulfilled at the saturation. Thus, by measuring the beam momentum dependence of $T$ on light nuclei it is possible to pin down the beam momentum dependence of the pion formation length (see, e.g., Figure 2a for ${ }^{12} \mathrm{C}$ target where the shapes of the $p_{\text {lab }}$ dependence for $\Delta M^{2}=0.7 \mathrm{GeV}^{2}$ and $1.4 \mathrm{GeV}^{2}$ significantly differ).
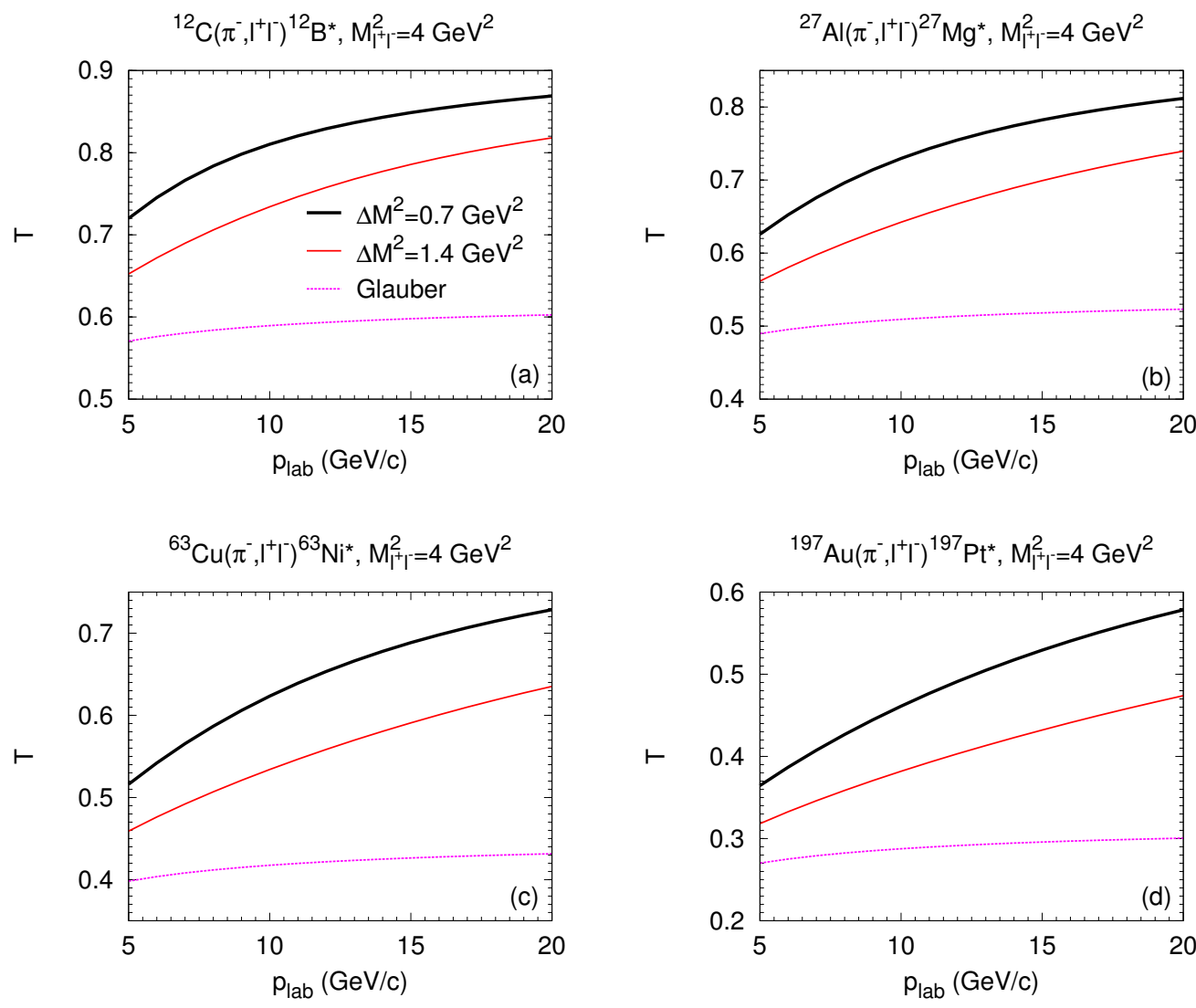

Figure 2. Transparency vs pion beam momentum for the $\left(\pi^{-}, l^{+} l^{-}\right)$reaction at fixed $M_{l^{+} l^{-}}^{2}=4 \mathrm{GeV}^{2}$ on the carbon (a), aluminum (b), copper (c), and gold (d) targets. Dashed (magenta) line-Glauber model, thick (black) and thin (red) solid line-QDM with $\Delta M^{2}=0.7$ and $1.4 \mathrm{GeV}^{2}$, respectively. 


\subsection{Large-Angle Pion Photoproduction}

The mechanism of the $\gamma n \rightarrow \pi^{-} p$ process significantly depends on the invariants $t=\left(p_{n}-p_{p}\right)^{2}$ and $u=\left(p_{n}-p_{\pi}\right)^{2}$. At $|t| \ll s / 2(|u| \ll s / 2)$ the photon converts to the $\rho$-meson long before the struck neutron and the process is dominated by the reggeized pion (nucleon) exchange. This regime is called the "resolved photon" (RP) regime which is based on the vector-dominance model [27]. With increasing $\min (|t|,|u|)$ the photon gradually looses its complex hadronic structure and interacts more and more like a bare electromagnetic state, i.e., the transition to the unresolved photon (UP) regime takes place. (In literature, unresolved photon is also often called the "direct" or "point-like" photon.) There is presently no theory that describes the both regimes simultaneously. However, one can use phenomenology to estimate $|t|$ at the transition. As follows from the asymptotic scaling law [28], in the UP regime the differential cross section $d \sigma / d t$ of the $\gamma N \rightarrow \pi N$ process should scale as $s^{-7}$ at $s \rightarrow \infty, t / s=$ const. The scaling $s^{-7}$ is observed at SLAC for $\gamma p \rightarrow \pi^{+} n$ at $\Theta_{c . m .}=90^{\circ}$ for $s \gtrsim 4 \mathrm{GeV}^{2}$ [29]. Thus, the value of $|t|$ at which the transition between the RP and UP regimes occurs can be estimated as $\sim s / 2 \sim 2 \mathrm{GeV}^{2}$. On the other hand, the onset of CT is also expected at about the same values of $|t|$ (see Figure 1 above). We expect then a complex interplay between the photon transparency (i.e., UP regime) and CT. How can one disentangle these two effects?

To this end we have calculated the nuclear transparency for the $A\left(\gamma, \pi^{-} p\right)$ process [30]:

$$
\begin{aligned}
T & =N^{-1} \int \mathrm{d}^{2} b \mathrm{~d} z \rho_{n}(b, z) \exp \left(-\sigma_{\gamma \mathrm{N}}^{\text {eff }} \int_{z-l_{\gamma}}^{z} \mathrm{~d} z^{\prime} \rho\left(b, z^{\prime}\right)\right. \\
& \left.-\int_{l_{r}}^{\infty} \mathrm{d} l \rho\left(b_{r}, l\right) \sigma_{\pi \mathrm{N}}^{\text {eff }}\left(p_{\pi}, l-l_{r}\right)-\int_{l_{r}^{\prime}}^{\infty} \mathrm{d} l^{\prime} \rho\left(b_{r^{\prime}}, l^{\prime}\right) \sigma_{p \mathrm{~N}}^{\text {eff }}\left(p_{p}, l^{\prime}-l_{r}^{\prime}\right)\right),
\end{aligned}
$$

where $z$ is along photon beam, $\rho_{n}(b, z)$ is the neutron density, $N$ is the total number of neutrons. $l$ and $l^{\prime}$ denote the coordinates along the linear trajectories of the outgoing pion and proton, respectively. The initial values and impact parameters are calculated using the spherical symmetry of the target nucleus: $l_{r}=\mathbf{r p}_{\pi} / p_{\pi}, b_{r}=\sqrt{r^{2}-l_{r}^{2}}, l_{r}^{\prime}=\mathbf{r p}_{p} / p_{p}, b_{r^{\prime}}=\sqrt{r^{2}-\left(l_{r}^{\prime}\right)^{2}}$, where $\mathbf{r} \equiv(\mathbf{b}, z)$. The effective photon-nucleon cross section, $\sigma_{\gamma \mathrm{N}}^{\text {eff }}$, accounts for the absorption of the intermediate $\rho$-meson in nuclear medium. In the RP regime, the distance traveled by the $\rho$-meson is approximately given by the photon coherence length

$$
l_{\gamma}=\frac{2 p_{\mathrm{lab}}}{m_{\rho}^{2}}
$$

and we set $\sigma_{\gamma \mathrm{N}}^{\text {eff }}$ equal to the inelastic $\pi N$ cross section. In the UP regime, the absorption of the photon is totally neglected, i.e., $\sigma_{\gamma \mathrm{N}}^{\text {eff }}=0$. The effective pion-nucleon cross section $\sigma_{\pi \mathrm{N}}^{\text {eff }}$ is given by the QDM expression, Equation (5), with $M_{\mathrm{CT}}^{2}=\min (-t,-u)$. For simplicity, we apply Equation (5) with $n=3$ for the effective proton-nucleon cross section $\sigma_{p \mathrm{~N}}^{\text {eff }}$ with replacement $\sigma_{\pi N} \rightarrow \sigma_{p \mathrm{~N}}$ and assuming $l_{p}=l_{\pi}$ for equal momenta of the proton and pion.

Figure 3 displays the nuclear transparency calculated assuming the UP and RP regimes, but disregarding CT. In the RP regime, the nuclear absorption is stronger due to the large $\rho N$ cross-section. However, in both regimes the nuclear transparency shows up a rather flat behavior as a function of beam momentum.

In contrast, as shown in Figure 4, the effect of CT is the increase of the nuclear transparency with

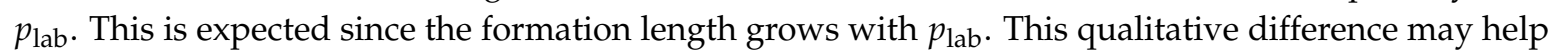
to disentangle the transition to the photon transparency from the onset of CT. 


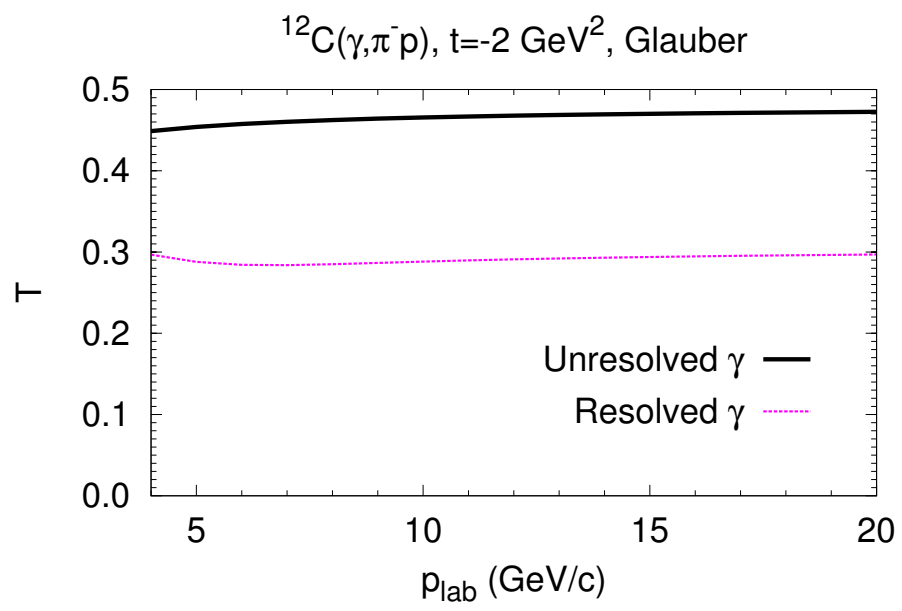

Figure 3. Transparency for the ${ }^{12} \mathrm{C}\left(\gamma, \pi^{-} p\right)$ semiexclusive process at $t=-2 \mathrm{GeV}^{2}$ vs photon beam momentum. Calculations for the unresolved photon (UP) and resolved photon (RP) regimes are shown by the solid (black) and dashed (magenta) line, respectively.

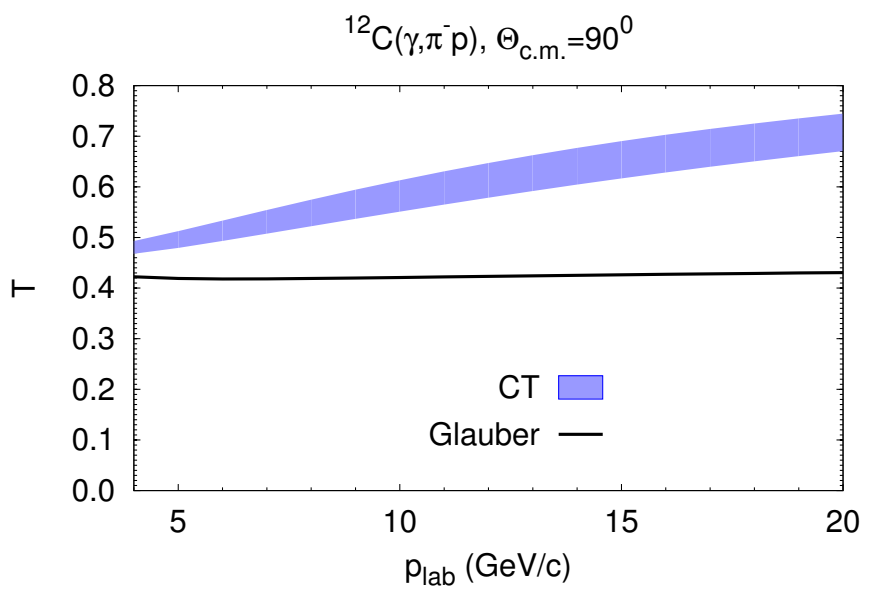

Figure 4. Transparency for the ${ }^{12} \mathrm{C}\left(\gamma, \pi^{-} p\right)$ semiexclusive process at $\Theta_{c . m .}=90^{\circ}$ vs. photon beam momentum. The band and solid line correspond to the QDM and Glauber model calculations, respectively. The upper (lower) boundary of the band is given by $\Delta M^{2}=0.7$ (1.1) $\mathrm{GeV}^{2}$.

\section{High-Energy Virtual-Photon-Nucleus Reactions}

The space-time scale of hadronization in high-energy $\gamma^{*} A$ DIS reactions should also be dominated by the hadron formation length that has a similar dependence on the hadron momentum as in exclusive processes, see Equation (3). Due to CT, during the formation stage pre-hadrons interact with nucleons with reduced strength. This picture is supported, in particular, by the GiBUU calculations of hadron attenuation at HERMES and EMC [17]. Hadron formation can also be tested by studying the production of low-energy neutrons from the decay of excited nuclear remnant. This has been initiated by the E665 experiment at Fermilab [31], where the neutrons with energy below $10 \mathrm{MeV}$ produced in $\mu^{-}$DIS at 470 $\mathrm{GeV}$ off $\mathrm{H}, \mathrm{D}, \mathrm{C}, \mathrm{Ca}$, and $\mathrm{Pb}$ targets have been detected. The main motivation was that the nucleus may serve as a "microcalorimeter" for high-energy hadrons: the excitation energy of the residual nucleus grows with the number of holes (wounded nucleons) and can be measured by the number of emitted low-energy neutrons. The first theoretical analysis of the E665 data performed in ref. [32] has led to the surprising conclusion that the CT effects are much stronger than those expected based on formation length (3) and are rather consistent with the scenario when only particles with momenta below $\sim 1 \mathrm{GeV} / \mathrm{c}$ interact with the nuclear remnant. 
We applied the GiBUU model (see detailed description in ref. [20]) to study slow neutron production induced by the passage of the DIS products through the nucleus [33]. This model solves the coupled system of kinetic equations for the baryons $\left(N, N^{*}, \Delta, \Lambda, \Sigma \ldots\right)$, corresponding antibaryons $\left(\bar{N}, \bar{N}^{*}, \bar{\Delta}, \bar{\Lambda}, \bar{\Sigma} \ldots\right)$, and mesons $(\pi, K, \ldots)$ explicitly in time and six-dimensional phase space of particle position and momentum by using the method of test particles. The collision term includes two- and three-body particle collisions and resonance decays. High-energy elementary binary collisions $(\sqrt{s}>2.2,3.4$ and $2.38 \mathrm{GeV}$ for meson-baryon, baryon-baryon, and antibaryon-baryon collisions, respectively) are simulated by the PYTHIA and (for antibaryon-baryon collisions only) FRITIOF models, while the low-energy ones are simulated my the Monte-Carlo method using empirical cross sections. Between collisions, the particles propagate along curved trajectories described by the Hamiltonian equations of motion in the non-relativistic Skyrme-like- and, optionally, relativistic (non-linear Walecka model) mean fields. (In the present calculations of DIS we apply the relativistic mean field NL3 of ref. [34]. We checked that using the medium (incompressibility $K=290 \mathrm{MeV}$ ) momentum-dependent Skyrme-like interaction (see Table 1 in ref. [20]) leads to practically indistinguishable results for neutron spectra. The pA and AA collisions were calculated in the cascade mode disregarding mean-field potentials.) In calculations, we applied the following alternative prescriptions for the pre-hadron-nucleon effective interaction cross section: (i) Time-dependent, based on the production $\left(t_{\text {prod }}\right)$ and formation $\left(t_{\text {form }}\right)$ times (see ref. [16], used as default in GiBUU) favored by the analysis of hadron attenuation at HERMES and EMC [17]:

$$
\sigma_{\text {eff }}(t) / \sigma_{0}=X_{0}+\left(1-X_{0}\right) \frac{t-t_{\text {prod }}}{t_{\text {form }}-t_{\text {prod }}}
$$

where $X_{0}=r_{\text {lead }} a / Q^{2}, a=1 \mathrm{GeV}^{2}, r_{\text {lead }}$ - the ratio of the number of leading quarks to the total number of quarks in the pre-hadron. (ii) Time-dependent, based on the QDM [6]:

$$
\sigma_{\text {eff }}(t) / \sigma_{0}=X_{0}+\left(1-X_{0}\right) \frac{c\left(t-t_{\text {hard }}\right)}{l_{h}},
$$

where $t_{\text {hard }}$ is the time of hard interaction (collision time instant), and the formation length is given by Equation (2) with $\Delta M^{2}=0.7 \mathrm{GeV}^{2}$. Note that the arguments leading to the initial size $\sim 1 / \mathrm{Q}$ may not be applicable as we are dealing with inclusive process here. Thus, for simplicity we set $X_{0}=0$. (iii) Momentum cutoff:

$$
\sigma_{\text {eff }} / \sigma_{0}=\Theta\left(p_{\text {cut }}-p_{h}\right), \quad p_{\text {cut }} \sim 1-2 \mathrm{GeV} / \mathrm{c} .
$$

Cascade of the interactions of DIS products in the nucleus leads to the direct emission of fast particles, including neutrons, and to the hole excitations of the nuclear residue. In order to describe the evaporation of slow neutrons from the excited nuclear residue, we applied the SMM [35,36]. The mass number $A_{\text {res }}$, charge number $Z_{\text {res }}$, excitation energy $E_{\text {res }}^{*}$ and momentum $\mathbf{p}_{\text {res }}$ of the nuclear residue were determined from GiBUU at the end of the time evolution $\left(t_{\max }=100 \mathrm{fm} / \mathrm{c}\right)$ and used as input for the SMM.

Figure 5 displays the calculated energy spectrum of neutrons in comparison with E665 data. The spectra are obtained under conditions $v>20 \mathrm{GeV}, Q^{2}>0.8 \mathrm{GeV}^{2}$ that select DIS events $(v$ is the energy of virtual photon in the target nucleus frame). One can see that almost all neutrons below $1 \mathrm{MeV}$ are statistically evaporated. The sensitivity to the model of hadron formation presents for $E_{n}>5 \mathrm{MeV}$. More restrictive conditions for the FSI of hadrons lead to smaller multiplicity of neutrons, mainly due to smaller excitation energy of the nuclear residue. The data can be only described with very strong restriction on the FSI $\left(p_{\text {cut }}=1 \mathrm{GeV} / \mathrm{c}\right)$, in agreement with earlier calculations [32]. 


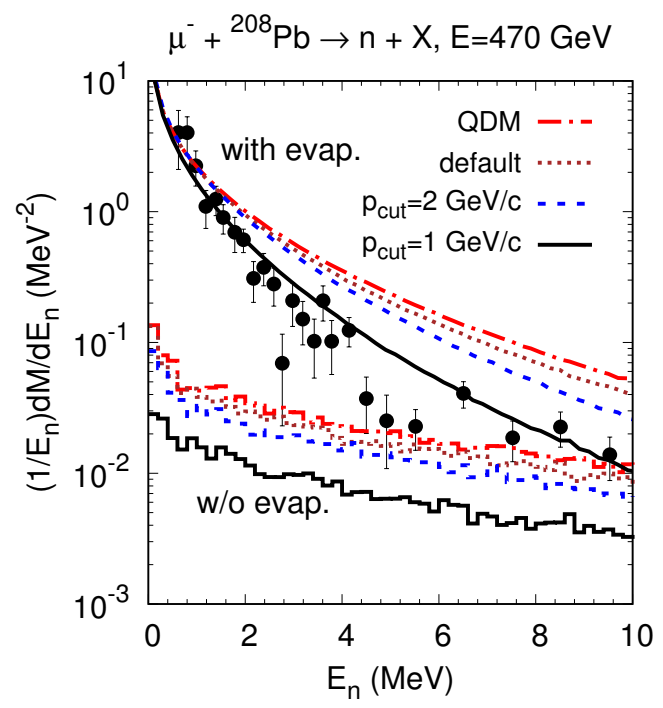

Figure 5. Energy spectrum of emitted neutrons in $\mu^{-}+{ }^{208} \mathrm{~Pb}$ deep inelastic scattering (DIS) at 470 $\mathrm{GeV}$. Different lines correspond to different prescriptions for the hadron formation: dash-dotted (red) line-QDM calculation; dotted (brown) line-Giessen Boltzmann-Uehling-Uhlenbeck (GiBUU) default; dashed (blue) line—cutoff momentum $2 \mathrm{GeV} / \mathrm{c}$; solid (black) line—cutoff momentum $1 \mathrm{GeV} / \mathrm{c}$. Upper (lower) lines are calculated with (without) adding evaporated neutrons from the nuclear residue. Experimental data are from ref. [31].

Various scenarios for hadron formation can be tested in ultraperipheral collisions (UPCs) of heavy ions. In such processes, the quasireal photons are emitted coherently by the entire nucleus [37] and get absorbed by another nucleus. The maximal longitudinal momentum of the photon in the c.m. frame of colliding nuclei (collider lab. frame) is determined by the inverse radius of the Lorentz-contracted nucleus:

$$
k_{L}^{\max } \simeq \frac{\gamma_{L}}{R_{A}}
$$

where $\gamma_{L}$ is the Lorentz factor. For symmetric colliding system in the rest frame of the target nucleus the maximum photon momentum is expressed as follows:

$$
k^{\max }=\gamma_{L} 2 k_{L}^{\max } \simeq \frac{2 \gamma_{L}^{2}}{R_{A}} .
$$

Table 1 summarizes the parameters of the symmetric UPCs at RHIC and the LHC. ( $W$ is the $\gamma N$ c.m. energy.) It is clear that using UPCs at these colliders one can study photon-nucleus interactions in the energy region never reachable so far and address the physics of hadronization in nuclear medium.

Table 1. Parameters of ultraperipheral collisions (UPCs) $\mathrm{Au}+\mathrm{Au}$ at RHIC and $\mathrm{Pb}+\mathrm{Pb}$ at the LHC.

\begin{tabular}{ccccc}
\hline & $\sqrt{s_{N N}}(\mathrm{TeV})$ & $\gamma_{L}$ & $k^{\mathrm{max}}(\mathrm{TeV} / \mathrm{c})$ & $W(\mathrm{GeV})$ \\
\hline RHIC & 0.2 & 106 & 0.642 & 34.7 \\
LHC & 5.5 & 2931 & 477 & 946 \\
\hline
\end{tabular}

We will focus on the photon-gluon interaction producing two jets: $\gamma^{*} g \rightarrow \bar{q} q$. The LC momentum fraction of the gluon is

$$
x_{g}=\frac{Q^{2}+M_{q \bar{q}}^{2}}{2 P q} \simeq x+\frac{M_{q \bar{q}}^{2}}{W^{2}}
$$


where $P$ and $q$ are the four-momenta of the struck nucleon and virtual photon, respectively, $W^{2}=(P+q)^{2}$, and $M_{\bar{q} q}$ is the invariant mass of the dijet. In the last step of (14) we assumed small $Q^{2}$. For the typical setting at the LHC [38]:

$$
M_{\bar{q} q} \simeq \mid p_{t}\left(\text { jet }_{1}\right)|+| p_{t}\left(\text { jet }_{2}\right) \mid \geq 40 \mathrm{GeV} .
$$

This condition eliminates $x_{g}$ in the gluon shadowing region.

In the GiBUU program package, the initial hard interaction is simulated via the PYTHIA model that can only describe a virtual photon emitted by the scattered lepton, $l \rightarrow l^{\prime} \gamma^{*}$. Moreover, the events with two high- $p_{t}$ jets are very rare. Thus, we rather rely on the inclusive set of PYTHIA events with fixed Bjorken $x=(40 \mathrm{GeV})^{2} / W^{2}$. It is clear from Equation (14) that this will produce the same lower limit on $x_{g}$ and, therefore, the same fragmentation pattern of the nucleon as in the case of the dijet production by the direct photon. This is important since the nucleon debris largely determine the production of slow particles.

Figure 6 shows the transverse momentum spectra of neutrons emitted in the hard virtual photon collisions with lead target in the fixed kinematics (a), and with lead and gold targets in different kinematics (b). The spectra are calculated with condition $x_{F}>0.1$ [39] which guaranties that the neutrons longitudinal momenta are directed along the target nucleus momentum in the collider laboratory frame. The Feynman variable $x_{F}$ is expressed as

$$
x_{F}=\frac{E-p^{z}}{\left(E_{A}-p_{A}^{z}\right) / A},
$$

where $E\left(E_{A}\right)$ and $p^{z}\left(p_{A}^{z}\right)$ are the particle (target nucleus) energy and the longitudinal component of momentum, respectively. The neutron spectra at $p_{t}=100-200 \mathrm{MeV} / \mathrm{c}$ show up a strong sensitivity to the hadron formation model. However, the photon kinematics has practically no influence. Thus, folding with actual photon flux is not expected to change significantly the neutron $p_{t}$-spectrum. Note that also the choice of the nuclear target (lead or gold) practically does not change the results.
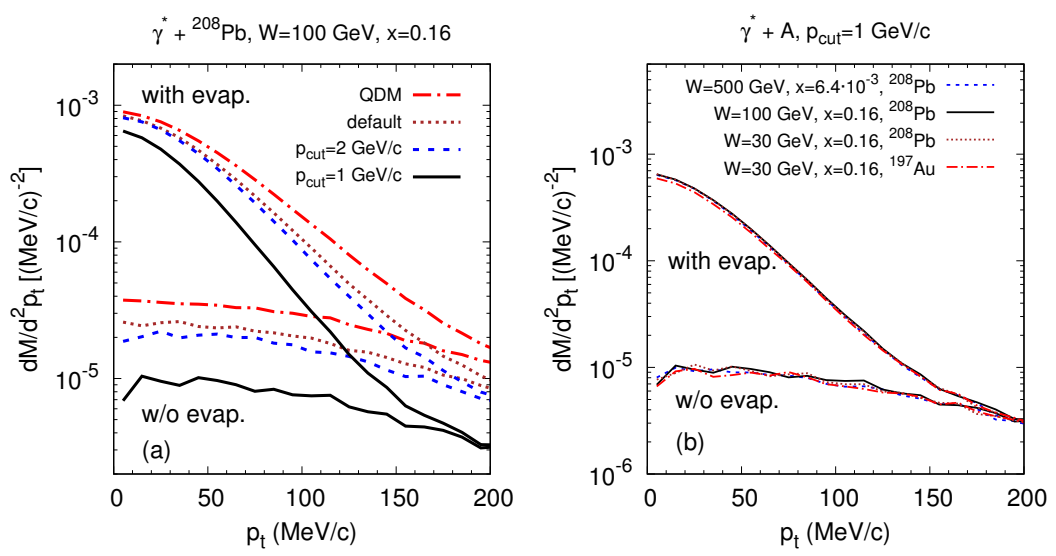

Figure 6. Neutron transverse momentum spectra for $\gamma^{*}+$ nucleus deep inelastic collisions. (a) Spectra for fixed photon kinematics $W=100 \mathrm{GeV}, x=0.16$ on the ${ }^{208} \mathrm{~Pb}$ target with different prescriptions for hadron formation (line notations are the same as in Figure 5). Upper (lower) lines show calculations with (without) statistical evaporation. (b) Spectra for the different photon kinematics and nuclear targets as indicated calculated with $p_{\text {cut }}=1 \mathrm{GeV} / \mathrm{c}$.

NICA allows to study the UPCs too. In Table 2 we provide the estimates of the parameters of the maximum photon momentum and $\gamma N$ c.m. energy reachable in $\mathrm{Au}+\mathrm{Au}$ and $\mathrm{p}+\mathrm{Au}$ collisions. For the latter, the photon can be emitted either by the gold nucleus or by the proton (we assume proton radius of $0.6 \mathrm{fm}$ ). Correspondingly, either $\gamma p$ or $\gamma \mathrm{Au}$ collisions are considered. 
In the $\mathrm{Au}+\mathrm{Au}$ UPCs one can study the baryon resonance excitation in nuclear medium and perform studies complementary to the JLab program. In the $\mathrm{p}+\mathrm{Au}$ UPCs with photon emitted by the proton we enter in the regime where the study of hard $\mathrm{pQCD}$ processes such as $J / \psi$ production and large-angle scattering become possible.

Table 2. Parameters of UPCs $\mathrm{Au}+\mathrm{Au}$ and $\mathrm{p}+\mathrm{Au}$ at NICA.

\begin{tabular}{ccccc}
\hline & $\sqrt{s_{N N}}(\mathrm{GeV})$ & $\gamma_{L}$ & $k^{\max }(\mathrm{GeV} / \mathrm{c})$ & $W(\mathrm{GeV})$ \\
\hline $\mathrm{Au}+\mathrm{Au}$ & 11.0 & 5.9 & 1.9 & 2.1 \\
$\mathrm{p}+\mathrm{Au}, \gamma p$ & 17.2 & 9.2 & 4.7 & 3.1 \\
$\mathrm{p}+\mathrm{Au}, \gamma \mathrm{Au}$ & 17.2 & 9.2 & 55.2 & 10.2 \\
\hline
\end{tabular}

\section{Proton-Nucleus and Nucleus-Nucleus Collisions}

Hadron formation reduces the FSI of pre-hadrons and thus we expect that the rapidity and transverse momentum distributions of produced particles in $\mathrm{pA}$ - and AA collisions will be affected. In this exploratory study we do not separate particles in the nuclear interior from those emitted in free space. In the case of pA collisions the calculation is performed in the rest frame of the target nucleus, while heavy ion collisions are calculated in the c.m. frame of the colliding nuclei.

Figure 7 displays the rapidity distributions of $p, \pi$ and $\Lambda+\Sigma^{0}$ in $p+A u$ and central $A u+A u$ collisions at $\sqrt{s}_{N N}=11 \mathrm{GeV}$. Neglecting formation length (i.e., assuming that hadrons are instantly formed) results in the largest yields at the intermediate rapidities ( $y \simeq 1$ for $p+A u, y \simeq 0$ for $\mathrm{Au}+\mathrm{Au}$ ). Restricting the FSI of produced particles by the introduction of either finite formation length or momentum cutoff depletes the intermediate rapidity region. The default GiBUU formation method and the QDM give almost indistinguishable results for $\mathrm{p}+\mathrm{Au}$ collisions while for $\mathrm{Au}+\mathrm{Au}$ collisions the QDM gives somewhat less stopping and less pion production than the GiBUU-default. Applying the momentum cutoff leads to the strongest constraints on the FSI. For the $\mathrm{p}+\mathrm{Au}$ system, the resulting rapidity distributions become depleted at $y \simeq 1$ and enhanced at $y \simeq 4$. The two bumps at these two rapidities are populated by the products of the target and projectile fragmentation, respectively. For the $\mathrm{Au}+\mathrm{Au}$ system at $b=1 \mathrm{fm}$, the momentum cutoff leads to the transparency pattern, especially pronounced for protons and hyperons in calculation with $p_{\text {cut }}=1 \mathrm{GeV} / \mathrm{c}$.

Figure 8 shows the $p_{t}$ spectra of $p, \pi$ and $\Lambda+\Sigma^{0}$. The bump in the proton spectrum at low $p_{t}$ 's is due to the bound protons in the target nucleus. Elastic rescattering increases the transverse momenta of outgoing hadrons. (This effect has been also observed in calculations of large-angle $d(p, p p) n[40]$ and $d\left(\bar{p}, \pi^{-} \pi^{0}\right) p$ [41] exclusive processes.) Thus, reducing FSI due to hadron formation makes the spectra steeper at large $p_{t}$ (closer to the direct production in first-chance $\mathrm{NN}$ collisions.
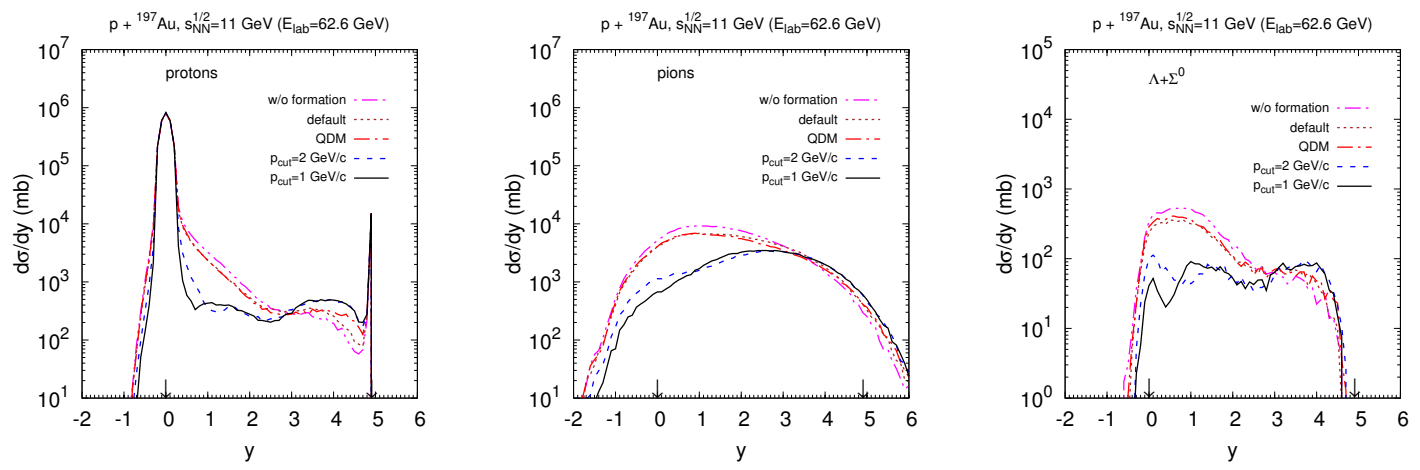

Figure 7. Cont. 

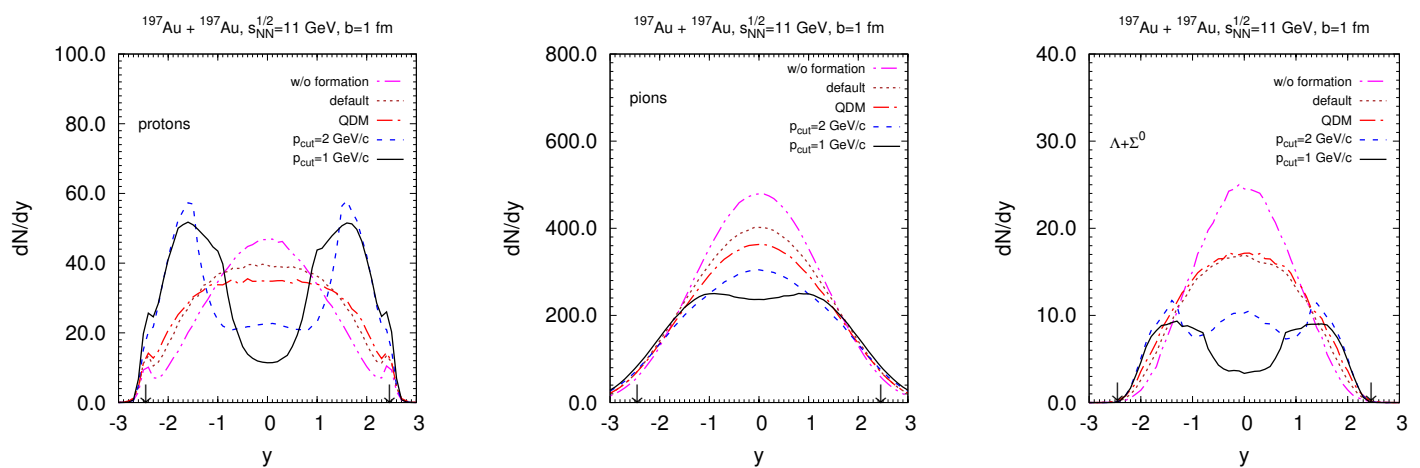

Figure 7. Rapidity spectra of protons, pions and neutral hyperons produced in minimum bias $\mathrm{p}+\mathrm{Au}$ collisions and central $(b=1 \mathrm{fm}) \mathrm{Au}+\mathrm{Au}$ collisions at $\sqrt{s}_{N N}=11 \mathrm{GeV}$. Different lines show calculations with different prescriptions for hadron formation (line notations are the same as in Figure 5). Arrows show rapidities of the projectile and target in the laboratory frame.
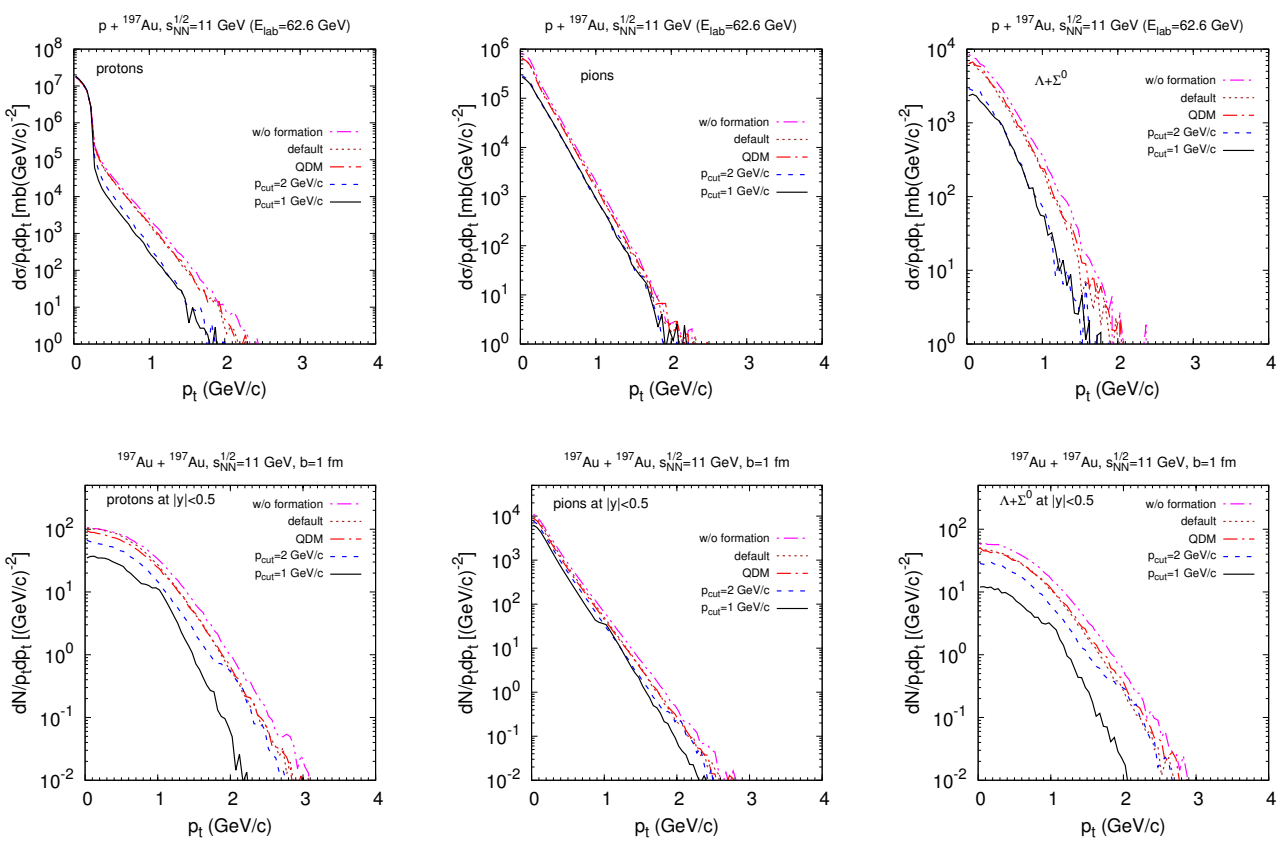

Figure 8. Transverse momentum spectra of protons, pions and neutral hyperons produced in minimum bias $\mathrm{p}+\mathrm{Au}$ collisions and central $(b=1 \mathrm{fm}) \mathrm{Au}+\mathrm{Au}$ collisions at $\sqrt{s}_{N N}=11 \mathrm{GeV}$. Different lines show calculations with different prescriptions for hadron formation (line notations are the same as in Figure 5). For the case of $\mathrm{Au}+\mathrm{Au}$ collisions, the rapidity cut $|y|<0.5$ has being applied for the spectra.

\section{Summary}

Color transparency is expected to be present in binary elementary reactions $a b \rightarrow c d$ with large scale $\gg 1 \mathrm{GeV}^{2}$ given by either $\min (|t|,|u|)$ or the (invariant mass) $)^{2}$ of one of participating particles. It is also expected that channels with mesons in the initial and/or final state are most promising for the observation of CT than pure baryonic processes since a $\bar{q} q$ pair is easier "squeezable" to PLC than a $q q q$ triple.

In this work we discussed the results of the Glauber and QDM calculations for the following semiexclusive reactions: $A\left(e, e^{\prime} \pi^{+}\right)$, pionic Drell-Yan process $A\left(\pi^{-}, l^{-} l^{+}\right)$with $M_{l^{-} l^{+}}^{2} \simeq 4 \mathrm{GeV}^{2}$, and large-angle pion photoproduction $A\left(\gamma, \pi^{-} p\right)$. For these three reactions, strong CT effects are predicted. In the first reaction, CT has been already observed at JLab. The second reaction is suggested to be studied at J-PARC. The third reaction provides also an additional opportunity to study 
the transition to the so-called photon transparency, i.e., the transition from resolved to unresolved (direct) photon with increasing $|t|$. This effect may interfere with CT and needs to be studied in more detail in the future.

CT-like behavior should also persist in inclusive reactions on nuclei at high energies, such as DIS, pA and AA collisions since they are governed by channels with large momentum transfer (large particle multiplicities). In these channels, the FSI is reduced due to a finite hadron formation length resulting in less secondary particles production and less deceleration of pre-hadrons produced in a primary hard collision. We have discussed slow neutron production in hard $\gamma^{*} A$ interactions. The hadronization dynamics in these processes can be probed by slow neutrons by using ultraperipheral collisions at the LHC and RHIC.

We have finally discussed proton, pion and neutral hyperon production in $\mathrm{pA}$ and AA collisions in the NICA regime. It is demonstrated, that the rapidity and transverse momentum spectra are quite sensitive to the assumptions on the hadron formation model. The effect of hadron formation may influence the formation and equilibration of the resonance matter [42] in the central region of the colliding system. Thus, the studies of hadron formation are complementary to the studies of the nuclear equation of state in heavy ion collisions.

Author Contributions: Investigation, A.L. and M.S. All authors have read and agreed to the published version of the manuscript.

Funding: This research was funded by HIC for FAIR within the framework of the Hessian LOEWE program, by U.S. Department of Energy, Office of Science, Office of Nuclear Physics, under Award No. DE-FG02-93ER40771, and by the German Federal Ministry of Education and Research (BMBF), Grant No. 05P18RGFCA.

Acknowledgments: The support of the Frankfurt Center for Scientific Computing is gratefully acknowledged.

Conflicts of Interest: The authors declare no conflict of interest.

\section{References}

1. Frankfurt, L.; Radyushkin, A.; Strikman, M. Interaction of small size wave packet with hadron target. Phys. Rev. D 1997, 55, 98-104, doi:10.1103/PhysRevD.55.98. [CrossRef]

2. Dutta, D.; Hafidi, K.; Strikman, M. Color transparency: Past, present and future. Prog. Part. Nucl. Phys. 2013, 69, 1-27, doi:10.1016/j.ppnp.2012.11.001. [CrossRef]

3. Aitala, E.M.; Amato, S.; Anjos, J.C.; Appel, J.A.; Ashery, D.; Banerjee, S.; Bediaga, I.; Blaylock, G.; Bracker, S.B.; Burchat, P.R.; et al. Observation of color transparency in diffractive dissociation of pions. Phys. Rev. Lett. 2001, 86, 4773-4777, doi:10.1103/PhysRevLett.86.4773. [CrossRef]

4. Frankfurt, L.; Miller, G.A.; Strikman, M. Coherent nuclear diffractive production of mini-jets: Illuminating color transparency. Phys. Lett. B 1993, 304, 1-7, doi:10.1016/0370-2693(93)91390-9. [CrossRef]

5. Frankfurt, L.; Miller, G.A.; Strikman, M. Coherent QCD phenomena in the coherent pion nucleon and pion nucleus production of two jets at high relative momenta. Phys. Rev. D 2002, 65, 094015, doi:10.1103/PhysRevD.65.094015. [CrossRef]

6. Farrar, G.; Liu, H.; Frankfurt, L.; Strikman, M. Transparency in nuclear quasiexclusive processes with large momentum transfer. Phys. Rev. Lett. 1988, 61, 686-689, doi:10.1103/PhysRevLett.61.686. [CrossRef] [PubMed]

7. Larson, A.; Miller, G.A.; Strikman, M. Pionic color transparency. Phys. Rev. C 2006, 74, 018201, doi:10.1103/PhysRevC.74.018201. [CrossRef]

8. Clasie, B.; Qian, X.; Arrington, J.; Asaturyan, R.; Benmokhtar, F.; Boeglin, W.; Bosted, P.; Bruell, A.; Christy, M.E.; Chudakov, E.; Cosyn, W.; et al. Measurement of nuclear transparency for the A(e, e-prime' pi+) reaction. Phys. Rev. Lett. 2007, 99, 242502, doi:10.1103/PhysRevLett.99.242502. [CrossRef] [PubMed]

9. El Fassi, L.; Zana, L.; Hafidi, K.; Holtrop, M.; Mustapha, B.; Brooks, W.K.; Hakobyan, H.; Zheng, X.; Adhikari, K.P.; Adikaram, D.; et al. Evidence for the onset of color transparency in $\rho^{0}$ electroproduction off nuclei. Phys. Lett. 2012, B712, 326-330, doi:10.1016/j.physletb.2012.05.019. [CrossRef]

10. Brodsky, S.J. Proceedings of the 13th International Symposium on Multiparticle Dynamics; Kittel, W., Metzger, W., Stergiou, A., Eds.; World Scientific: Singapore, 1982; p. 963. 
11. Mueller, A.H. Proceedings of the 17 th Rencontres de Moriond; Tran Thanh Van, J., Ed.; Editions Frontieres: Gif-sur-Yvette, France, 1982; Volume I, p. 13.

12. Leksanov, A.; Alster, J.; Asryan, G.; Averichev, Y.; Barton, D.; Baturin, V.; Bukhtoyarova, N.; Carroll, A.; Heppelmann, S.; Kawabata, T.; et al. Energy dependence of nuclear transparency in C(p, 2p) scattering. Phys. Rev. Lett. 2001, 87, 212301, doi:10.1103/PhysRevLett.87.212301. [CrossRef] [PubMed]

13. Brodsky, S.J.; de Teramond, G.F. Spin correlations, QCD color transparency and heavy quark thresholds in proton proton scattering. Phys. Rev. Lett. 1988, 60, 1924, doi:10.1103/PhysRevLett.60.1924. [CrossRef] [PubMed]

14. Ralston, J.P.; Pire, B. Fluctuating proton size and oscillating nuclear transparency. Phys. Rev. Lett. 1988, 61, 1823, doi:10.1103/PhysRevLett.61.1823. [CrossRef] [PubMed]

15. Kopeliovich, B.Z.; Nemchik, J.; Predazzi, E.; Hayashigaki, A. Nuclear hadronization: Within or without? Nucl. Phys. A 2004, 740, 211-245, doi:10.1016/j.nuclphysa.2004.04.110. [CrossRef]

16. Gallmeister, K.; Falter, T. Space-time picture of fragmentation in PYTHIA/JETSET for HERMES and RHIC. Phys. Lett. B 2005, 630, 40-48, doi:10.1016/j.physletb.2005.08.135. [CrossRef]

17. Gallmeister, K.; Mosel, U. Time dependent hadronization via HERMES and EMC data consistency. Nucl. Phys. A 2008, 801, 68-79, doi:10.1016/j.nuclphysa.2007.12.009. [CrossRef]

18. Bass, S.A.; Belkacem, M.; Bleicher, M.; Brandstetter, M.; Bravina, L.; Ernst, C.; Gerland, L.; Hofmann, M.; Hofmann, S.; Konopka, J.; et al. Microscopic models for ultrarelativistic heavy ion collisions. Prog. Part. Nucl. Phys. 1998, 41, 255-369, doi:10.1016/S0146-6410(98)00058-1. [CrossRef]

19. Cassing, W.; Bratkovskaya, E.L. Hadronic and electromagnetic probes of hot and dense nuclear matter. Phys. Rept. 1999, 308, 65-233, doi:10.1016/S0370-1573(98)00028-3. [CrossRef]

20. Buss, O.; Gaitanos, T.; Gallmeister, K.; van Hees, H.; Kaskulov, M.; Lalakulich, O.; Larionov, A.B.; Leitner, T.; Weil, J.; Mosel, U. Transport-theoretical description of nuclear reactions. Phys. Rept. 2012, 512, 1-124, doi:10.1016/j.physrep.2011.12.001. [CrossRef]

21. Kaskulov, M.M.; Gallmeister, K.; Mosel, U. Deeply inelastic pions in the exclusive reaction p(e, e' pi+)n above the resonance region. Phys. Rev. D 2008, 78, 114022, doi:10.1103/PhysRevD.78.114022. [CrossRef]

22. Collins, J.C.; Frankfurt, L.; Strikman, M. Factorization for hard exclusive electroproduction of mesons in QCD. Phys. Rev. D 1997, 56, 2982-3006, doi:10.1103/PhysRevD.56.2982. [CrossRef]

23. Berger, E.R.; Diehl, M.; Pire, B. Probing generalized parton distributions in pi N $\rightarrow 1+1-$ N. Phys. Lett. B 2001, 523, 265-272, doi:10.1016/S0370-2693(01)01345-4. [CrossRef]

24. Goloskokov, S.V.; Kroll, P. The exclusive limit of the pion-induced Drell-Yan process. Phys. Lett. B 2015, 748, 323-327, doi:10.1016/j.physletb.2015.07.016. [CrossRef]

25. Anikin, I.V.; Batzell, N.; Boer, M.; Boussarie, R.; Braun, V.M.; Brodsky, S.J.; Camsonne, A.; Chang, W.C.; Colaneri, L.; Dobbs, S.; et al. Nucleon and nuclear structure through dilepton production. Acta Phys. Polon. B 2018, 49, 741-784, doi:10.5506/APhysPolB.49.741. [CrossRef]

26. Larionov, A.B.; Strikman, M.; Bleicher, M. Color transparency in $\pi^{-}$-induced dilepton production on nuclei. Phys. Rev. C 2016, 93, 034618, doi:10.1103/PhysRevC.93.034618. [CrossRef]

27. Bauer, T.H.; Spital, R.D.; Yennie, D.R.; Pipkin, F.M. The hadronic properties of the photon in high-energy interactions. Rev. Mod. Phys. 1978, 50, 261, doi:10.1103/RevModPhys.50.261. [CrossRef]

28. Brodsky, S.J.; Farrar, G.R. Scaling laws at large transverse momentum. Phys. Rev. Lett. 1973, 31, 1153-1156, doi:10.1103/PhysRevLett.31.1153. [CrossRef]

29. Anderson, R.L.; Gustavson, D.; Ritson, D.; Weitsch, G.A.; Halpern, H.J.; Prepost, R.; Tompkins, D.H.; Wiser, D.E. Measurements of exclusive photoproduction processes at large values of $\mathrm{t}$ and $\mathrm{u}$ from $4-\mathrm{GeV}$ to 7.5-GeV. Phys. Rev. D 1976, 14, 679, doi:10.1103/PhysRevD.14.679. [CrossRef]

30. Larionov, A.B.; Strikman, M. Exploring QCD dynamics in medium energy $\gamma$ A semiexclusive collisions. Phys. Lett. B 2016, 760, 753-758, doi:10.1016/j.physletb.2016.07.067. [CrossRef]

31. Adams, M.R.; Aïd, S.; Anthony, P.L.; Averill, D.A.; Baker, M.D.; Baller, B.R.; Banerjee, A.; Bhatti, A.A.; Bratzler, U.; Braun, H.M.; et al. Nuclear decay following deep inelastic scattering of $470-\mathrm{GeV}$ muons. Phys. Rev. Lett. 1995, 74, 5198-5201, doi:10.1103/PhysRevLett.74.5198, 10.1103/PhysRevLett.80.2020. [CrossRef]

32. Strikman, M.; Tverskoy, M.G.; Zhalov, M.B. Soft neutron production in DIS: A Window to the final state interactions. Phys. Lett. B 1999, 459, 37-42, doi:10.1016/S0370-2693(99)00627-9. [CrossRef] 
33. Larionov, A.B.; Strikman, M. Slow neutron production as a probe of nuclear transparency and hadron formation in high-energy $\gamma^{*} A$ reactions. arXiv 2018, arXiv:1812.08231.

34. Lalazissis, G.A.; König, J.; Ring, P. A new parameterization for the lagrangian density of relativistic mean field theory. Phys. Rev. C 1997, 55, 540-543, doi:10.1103/PhysRevC.55.540. [CrossRef]

35. Botvina, A.S.; Iljinov, A.S.; Mishustin, I.N.; Bondorf, J.P.; Donangelo, R.; Sneppen, K. Statistical simulation of the breakup of highly excited nuclei. Nucl. Phys. A 1987, 475, 663-686, doi:10.1016/0375-9474(87)90232-6. [CrossRef]

36. Bondorf, J.P.; Botvina, A.S.; Ilinov, A.S.; Mishustin, I.N.; Sneppen, K. Statistical multifragmentation of nuclei. Phys. Rept. 1995, 257, 133-221, doi:10.1016/0370-1573(94)00097-M. [CrossRef]

37. Baltz, A.J. The physics of ultraperipheral collisions at the LHC. Phys. Rept. 2008, 458, 1-171, doi:10.1016/j.physrep.2007.12.001. [CrossRef]

38. Aad, G.; Abbott, B.; Abdallah, J.; Abdinov, O.; Aben, R.; Abolins, M.; AbouZeid, O.S.; Abramowicz, H.; Abreu, H.; Abreu, R.; et al. Dijet production in $\sqrt{s}=7 \mathrm{TeV} p p$ collisions with large rapidity gaps at the ATLAS experiment. Phys. Lett. B 2016, 754, 214-234, doi:10.1016/j.physletb.2016.01.028. [CrossRef]

39. White, S.N. Very forward calorimetry at the LHC: Recent results from ATLAS. AIP Conf. Proc. 2011, 1350, 95-101, doi:10.1063/1.3601384. [CrossRef]

40. Frankfurt, L.L.; Piasetzky, E.; Sargsian, M.M.; Strikman, M.I. On the possibility to study color transparency in the large momentum transfer exclusive d (p, 2 p) n reaction. Phys. Rev. 1997, C56, 2752-2766, doi:10.1103/PhysRevC.56.2752. [CrossRef]

41. Larionov, A.B.; Strikman, M. Color transparency in $\bar{p} d \rightarrow \pi^{-} \pi^{0} p$ reaction. arXiv 2019, arXiv:1909.00379.

42. Ehehalt, W.; Cassing, W.; Engel, A.; Mosel, U.; Wolf, G. Resonance properties in nuclear matter. Phys. Rev. C 1993, 47, R2467-R2469, doi:10.1103/PhysRevC.47.R2467. [CrossRef]

(C) 2020 by the authors. Licensee MDPI, Basel, Switzerland. This article is an open access article distributed under the terms and conditions of the Creative Commons Attribution (CC BY) license (http:/ / creativecommons.org/licenses/by/4.0/). 